\title{
The Inhibition of the Components from Shengmai Injection towards UDP-Glucuronosyltransferase
}

\author{
Li-Peng Jiang, ${ }^{1}$ Jin Zhao, ${ }^{2}$ Yun-Feng Cao, ${ }^{3}$ Mo Hong, ${ }^{4,5}$ Dong-Xue Sun, \\ Xiao-Yu Sun, ${ }^{4,5}$ Jun Yin, ${ }^{2}$ Zhi-Tu Zhu, ${ }^{1}$ and Zhong-Ze Fang ${ }^{6}$ \\ ${ }^{1}$ The First Affiliated Hospital of Liaoning Medical University, Jinzhou, Liaoning 121001, China \\ ${ }^{2}$ School of Traditional Chinese Medicine, Shenyang Pharmaceutical University, Shenyang 110016, China \\ ${ }^{3}$ Key Laboratory of Contraceptives and Devices Research (NPFPC), Shanghai Engineer and Technology Research Center of \\ Reproductive Health Drug and Devices, Shanghai Institute of Planned Parenthood Research, Shanghai 200032, China \\ ${ }^{4}$ Joint Center for Translational Medicine, Dalian Institute of Chemical Physics, Chinese Academy of Sciences and \\ The First Affiliated Hospital of Liaoning Medical University, No. 457, Zhongshan Road, Dalian 116023, China \\ ${ }^{5}$ Joint Center for Translational Medicine, Dalian Institute of Chemical Physics, Chinese Academy of Sciences and \\ The Affiliated Zhongshan Hospital of Dalian University, No. 6, Jiefang Street, Zhongshan District, Dalian 116001, China \\ ${ }^{6}$ Department of Toxicology, School of Public Health, Tianjin Medical University, 22 Qixiangtai Road, \\ Heping District, Tianjin 300070, China
}

Correspondence should be addressed to Zhi-Tu Zhu; zzfang228@gmail.com and Zhong-Ze Fang; fangzhongze@tijmu.edu.cn

Received 8 August 2014; Revised 24 September 2014; Accepted 8 October 2014; Published 29 October 2014

Academic Editor: Yong Jiang

Copyright (C) 2014 Li-Peng Jiang et al. This is an open access article distributed under the Creative Commons Attribution License, which permits unrestricted use, distribution, and reproduction in any medium, provided the original work is properly cited.

\begin{abstract}
The mechanism of shengmai injection- (SMI-) related drug-drug interaction remains unclear. Evaluation of the inhibition potential of SMI's ingredients towards UDP-glucuronosyltransferases (UGTs) activity will provide a new insight to understand SMI-related drug-drug interaction. In vitro incubation system to model UGT reaction was used. Recombinant UGT isoforms-catalyzed 4-methylumbelliferone (4-MU) glucuronidation and UGT1A4-catalyzed trifluoperazine (TFP) glucuronidation reactions were employed to phenotype the inhibition profile of maidong's components towards the activity of UGT isoforms. Different inhibition potential of maidong's components towards various UGT isoforms was observed. Based on the inhibition kinetic investigation results, ophiopogonin D (OD) noncompetitively inhibited UGT1A6 and competitively inhibited UGT1A8, ophiopogonin $\mathrm{D}^{\prime}$ (OD') noncompetitively inhibited UGT1A6 and UGT1A10, and ruscorectal (RU) exhibited competitive inhibition towards UGT1A4. The inhibition kinetic parameters were calculated to be $20.6,40.1,5.3,9.0$, and $0.02 \mu \mathrm{M}$, respectively. In combination with our previous results obtained for the inhibition of UGT isoforms by ginsenosides and wuweizi components, the important SMI ingredients exhibiting strong inhibition towards UGT isoforms were highlighted. All the results obtained in the present study provide a new insight to understand SMI-related drug-drug interaction.
\end{abstract}

\section{Introduction}

Shengmai San, a well-known traditional Chinese herbal prescription recorded in Yixueqiyuan (Origins of Medicine), has been routinely employed to treat cardiovascular diseases for thousands of years in China [1]. Shengmai injection (SMI), developed on the basis of Shengmai San, has been clinically applied. SMI contains the following herbs: Renshen (Radix Ginseng; Ginseng), Maidong (Radix Ophiopogonis; Dwarf Lilyturf Tuber), and Wuweizi (Fructus schisandrae chinensis; Chinese Magnoliavine Fruit). The clinical safety of SMI has been speculated, and the adverse effects were detected in 36 patients taking SMI, including 17 male and 19 female [2]. The main adverse effect for patients taking SMI is anaphylactic reaction [2]. Additionally, clinical drug-drug interaction between SMI and warfarin was also reported [3].

UDP-glucuronosyltransferases (UGTs) are important metabolizing enzymes involved in the metabolism of many xenobiotics (e.g., propofol, zidovudine, etc.) and endogenous substances (e.g., bilirubin, bile acids, etc.) [4]. UGTs have 


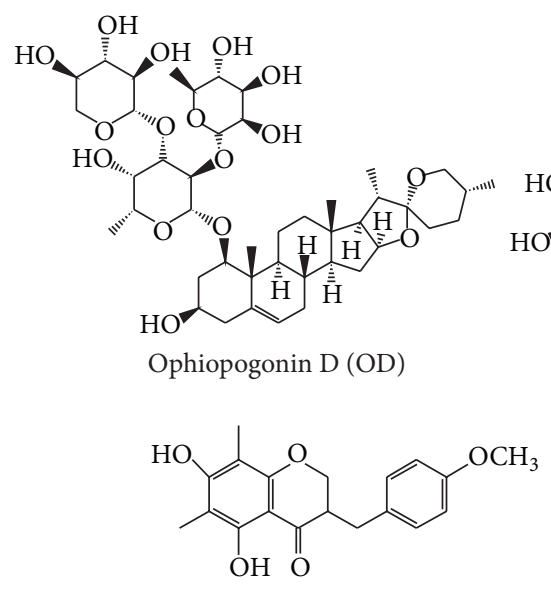

Methylophiopogonanone B (MB)

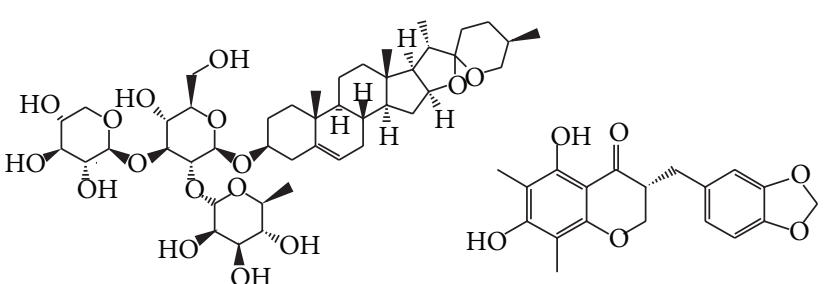

Ophiopogonin $\mathrm{D}^{\prime}\left(\mathrm{OD}^{\prime}\right) \quad$ Methylophiopogonanone A (MA)

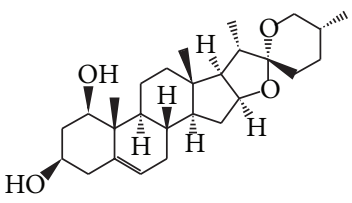

Ruscorectal (RU)

FIGURE 1: The structures of ophiopogonin D (OD), ophiopogonin $\mathrm{D}^{\prime}\left(\mathrm{OD}^{\prime}\right)$, methylophiopogonanone A (MA), methylophiopogonanone B $(\mathrm{MB})$, and ruscorectal (RU).

been demonstrated to exhibit expression in multiple tissues, mainly in liver and intestine. To date, approximately 117 members have been found in mammalian UGT gene superfamily, and they are divided into four families, UGT1, UGT2, UGT3, and UGT8. Inhibition of UGTs' activity can induce severe adverse effects. Indinavir, an important HIV therapeutic drug, has been reported to significantly induce the elevation of serum-unconjugated bilirubin through inhibiting UGT1A1-catalyzed bilirubin glucuronidation [5]. Sorafenibinduced UGT1A1 inhibition might also be an important reason for the elevation of serum bilirubin [6].

To date, the mechanism for SMI-related drug-drug interaction remains unclear. Evaluation of SMI's components' inhibitory potential towards UGTs isoforms will provide us with a new insight to elucidate the mechanism of SMIrelated drug-drug interaction. In our previous study, relevant work has been performed for ginseng and wuweizi's ingredients. Ginsenosides, the major components of ginseng, have been demonstrated to exhibit strong structure-dependent inhibition towards many UGT isoforms [7]. Additionally, deoxyschizandrin and schisantherin A, two important ingredients isolated from wuweizi, have been demonstrated to exhibit inhibition towards UGT1A3 [8].

All these results give us the data support and confidence to investigate the inhibition of maidong's components towards UGT isoforms. Using the combination of the present data and previous UGTs' inhibition data for ginsenosides and wuweizi lignans, the important SMI ingredients exhibiting strong inhibition towards UGT isoforms were highlighted.

\section{Materials and Methods}

2.1. Chemicals. 4-Methylumbelliferone (4-MU), 4-methylumbelliferone- $\beta$-D-glucuronide (4-MUG), Tris-HCl, 7-hydroxycoumarin, and uridine -5 -diphosphoglucuronic acid
(UDPGA) (trisodium salt) were purchased from SigmaAldrich (St. Louis, MO). Recombinant human UGT isoforms (UGT1A1, UGT1A3, UGT1A4, UGT1A6, UGT1A7, UGT1A8, UGT1A9, UGT1A10, UGT2B4, UGT2B7, and UGT2B15) expressed in baculovirus-infected insect cells were obtained from BD Gentest Corp. (Woburn, MA, USA). Ophiopogonin $\mathrm{D}(\mathrm{OD})$, ophiopogonin $\mathrm{D}^{\prime}\left(\mathrm{OD}^{\prime}\right)$, methylophiopogonanone A (MA), methylophiopogonanone B (MB), and ruscorectal (RU) were purchased from Sichuan Weikeqi Biotechnology Company (Chengdu, Sichuan, China). The purity of these compounds was above 95\%. All other reagents were of HPLC grade or of the highest grade commercially available.

2.2. Initial Screening of Maidong' Components Inhibition towards Recombinant UGTs Activity. 4-MU, the nonspecific probe substrate for all the UGT isoforms except UGT1A4, was used in the present study to initially screen the inhibition potential of maidong's components towards UGT isoforms as previously reported [7]. In brief, the incubation system (total volume $=200 \mu \mathrm{L}$ ) consisted of recombinant UGTs, $5 \mathrm{mM}$ UDPGA, $5 \mathrm{mM} \mathrm{MgCl}$, $50 \mathrm{mM}$ Tris- $\mathrm{HCl}$ ( $\mathrm{pH}=7.4$ ), and 4$\mathrm{MU}$ in the absence or presence of different concentrations of maidong's components. The compounds were dissolved in DMSO, and the final concentration of DMSO was below $0.5 \%$ $(\mathrm{v} / \mathrm{v})$. The used incubation time and protein concentration were previously determined to ensure the reaction rate within the linear range. The 4-MU concentration was equal to known $K_{m}$ or $S_{50}$ values for each UGT isoform [7]. After 5 min preincubation at $37^{\circ} \mathrm{C}$, the incubation reaction was initiated via adding UDPGA to the mixture system. The reactions were quenched by adding $100 \mu \mathrm{L}$ acetonitrile with 7hydroxycoumarin $(100 \mu \mathrm{M})$ as internal standard. Centrifuged at $20000 \times \mathrm{g}$ for $10 \mathrm{~min}$, an aliquot of supernatant was transferred to an autoinjector vial for HPLC analysis. The HPLC system (Shimadzu, Kyoto, Japan) contained a SCL-10A system controller, two LC-10AT pumps, a SIL-10A autoinjector, 

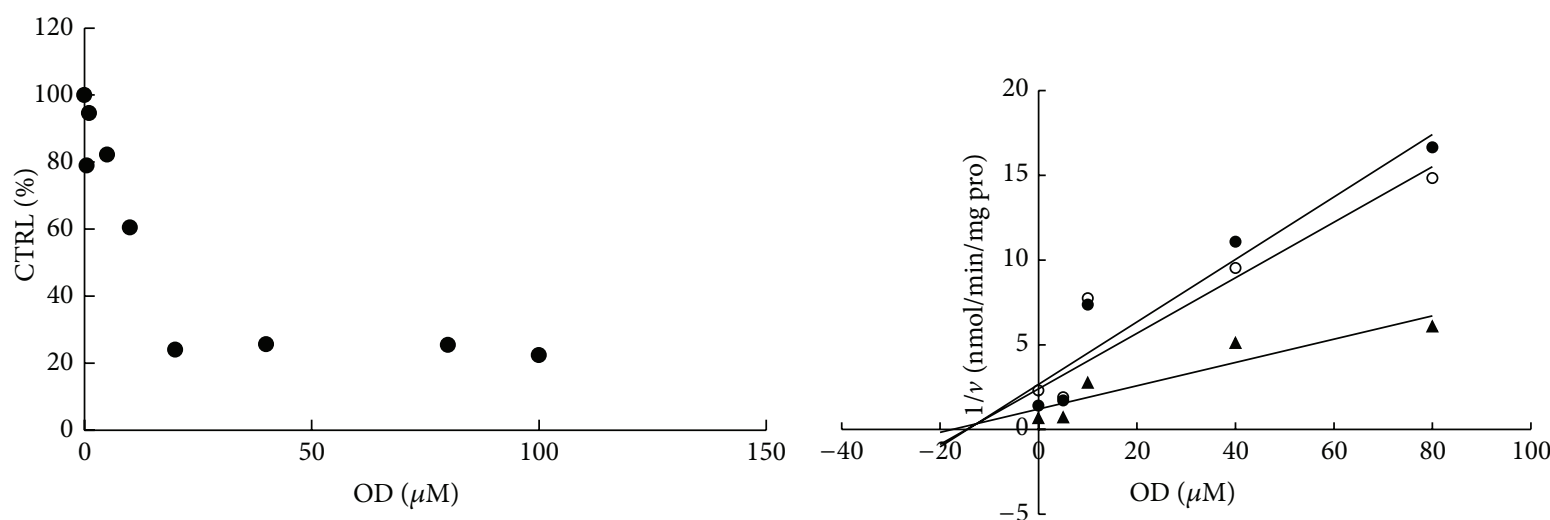

○ 4 -MU $30 \mu \mathrm{M}$

- $4-\mathrm{MU} 50 \mu \mathrm{M}$

\ $4-\mathrm{MU} 110 \mu \mathrm{M}$

(a)

(b)

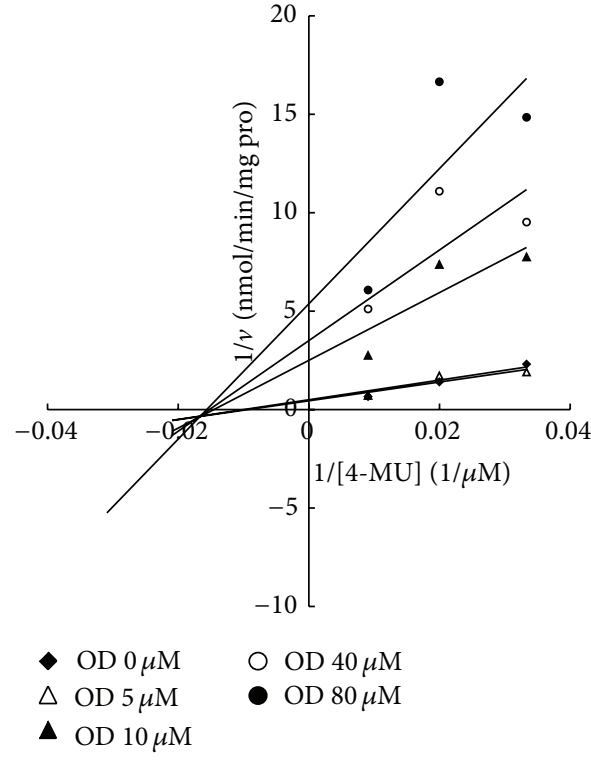

(c)

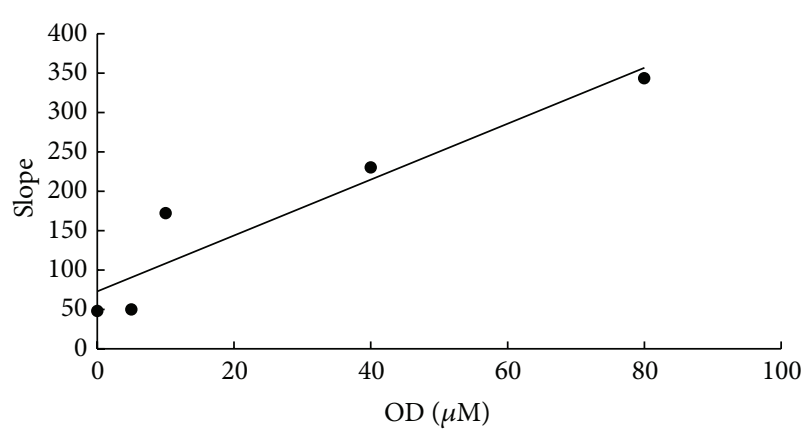

(d)

FIGURE 2: Determination of inhibition type and parameters $\left(K_{i}\right)$ of OD towards UGT1A6-catalyzed 4-MU glucuronidation reaction. (a) Dose-dependent inhibition of OD towards UGT1A6-catalyzed 4-MU glucuronidation. (b) Dixon plot of inhibition of OD towards UGT1A6catalyzed 4-MU glucuronidation. (c) Lineweaver-Burk plot of inhibition of OD towards UGT1A6-catalyzed 4-MU glucuronidation. (d) Second plot of inhibition of OD towards UGT1A6-catalyzed 4-MU glucuronidation. The data point represents the mean value of duplicate experiments.

and a SPD-10AVP UV detector. Chromatographic separation was carried out using a C18 column $(4.6 \times 200 \mathrm{~mm}, 5 \mu \mathrm{m}$, Kromasil) at a flow rate of $1 \mathrm{~mL} / \mathrm{min}$ and UV detector at $316 \mathrm{~nm}$. The mobile phase consisted of acetonitrile (A) and $\mathrm{H}_{2} \mathrm{O}$ containing $0.5 \%(\mathrm{v} / \mathrm{v})$ formic acid (B). The following gradient condition was used: 0-15 $\mathrm{min}, 95-40 \% \mathrm{~B} ; 15-20 \mathrm{~min}$, 10\% B; 20-30 $\mathrm{min}, 95 \% \mathrm{~B}$.

UGT1A4 exhibited low catalytic activity towards 4-MU glucuronidation. Therefore, UGT1A4-catalyzed trifluoperazine (TFP) glucuronidation was carried out to evaluate inhibition of maidong's components towards UGT1A4 activity. As previously reported [9], TFP ( $40 \mu \mathrm{M}$, near its $K_{m}$ value) was incubated with $0.1 \mathrm{mg} / \mathrm{mL}$ recombinant UGT1A4 for $20 \mathrm{~min}$ in the presence or absence of maidong's ingredients.

2.3. Inhibition Kinetic Analysis. To determine the inhibition kinetic type and parameters $\left(K_{i}\right)$, the reaction velocity $(V)$ was determined at multiple concentrations of maidong's components and 4-MU (or TFP). Dixon plot and LineweaverBurk plot were used to fit the data. The inhibition kinetic type was evaluated through determining the intersection point in the Dixon and Lineweaver-Burk plots. 


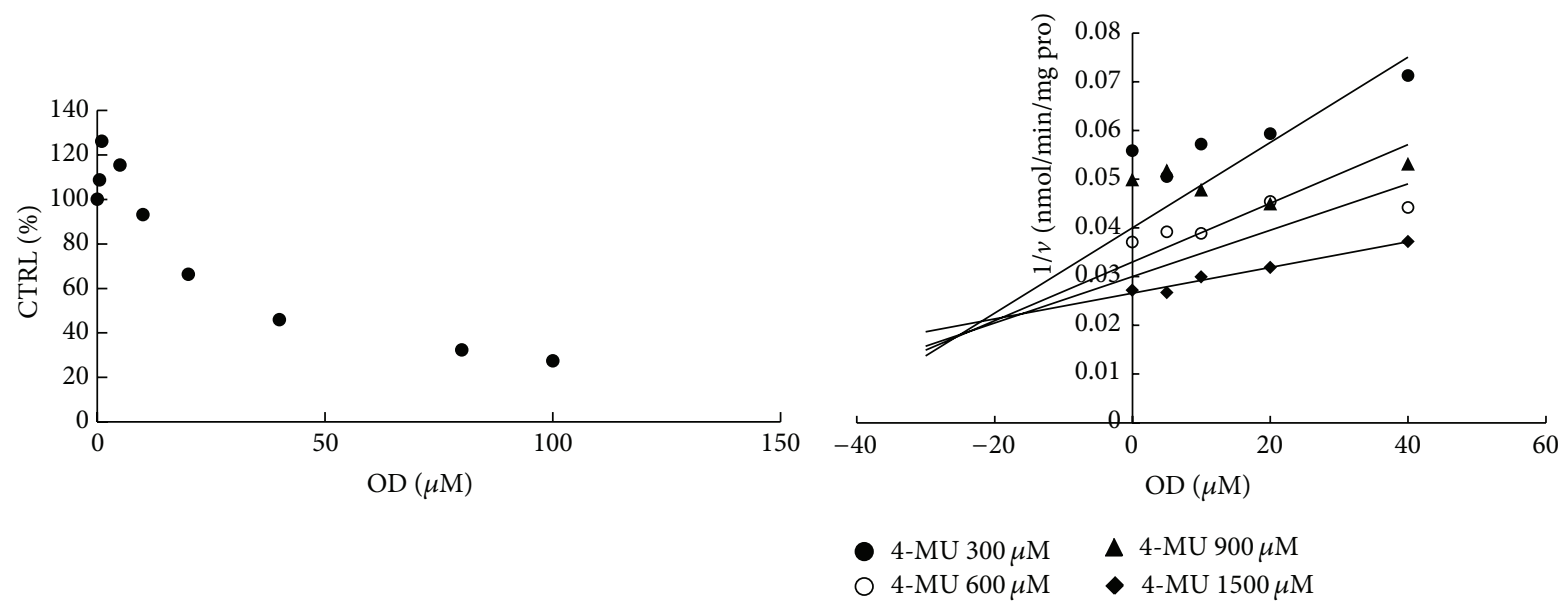

(a)

(b)

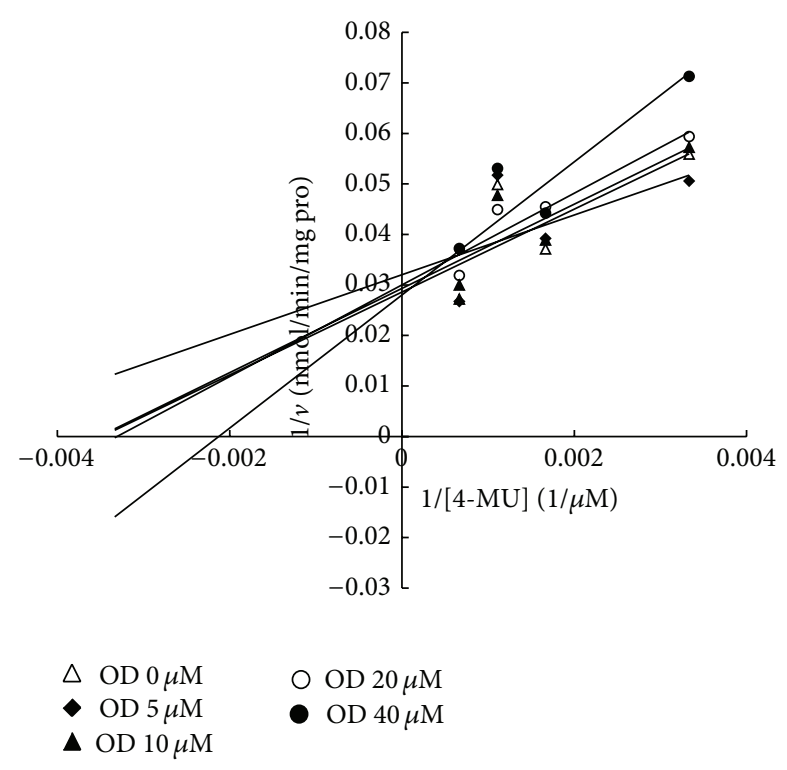

(c)

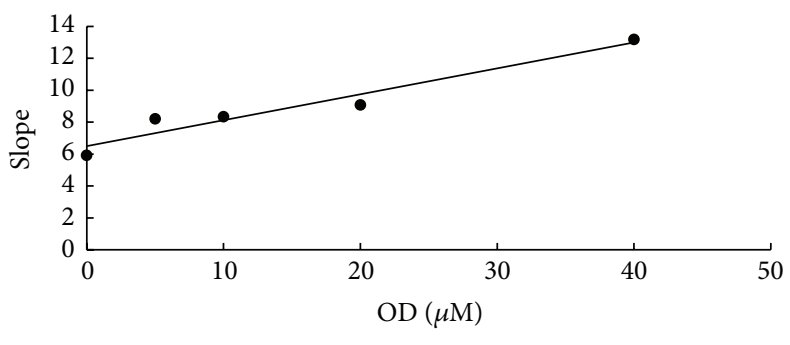

(d)

FIGURE 3: Determination of inhibition type and parameters $\left(K_{i}\right)$ of OD towards UGT1A8-catalyzed 4-MU glucuronidation reaction. (a) Dose-dependent inhibition of OD towards UGT1A8-catalyzed 4-MU glucuronidation. (b) Dixon plot of inhibition of OD towards UGT1A8catalyzed 4-MU glucuronidation. (c) Lineweaver-Burk plot of inhibition of OD towards UGT1A8-catalyzed 4-MU glucuronidation. (d) Second plot of inhibition of OD towards UGT1A8-catalyzed 4-MU glucuronidation. The data point represents the mean value of duplicate experiments.

Nonlinear regression was used to calculate the inhibition kinetic parameters $\left(K_{i}\right)$ according to the equations for competitive inhibition (1) and noncompetitive inhibition (2):

$$
\begin{gathered}
V=\frac{V_{\max } *[S]}{\left(K_{m} /\left(1+\left([I] / K_{i}\right)\right)+[S]\right)}, \\
V=\frac{V_{\max } *[S]}{\left(K_{m}+[S]\right) *\left(1+\left([I] / K_{i}\right)\right)},
\end{gathered}
$$

where the items are defined as follows: $V$ is the reaction velocity and $[S]$ and $[I]$ are the concentrations of substrate and inhibitor, respectively. $K_{m}$ value is the substrate concentration in which the velocity reached half of the maximum velocity $\left(V_{\max }\right)$ of the reaction. $K_{i}$ value is the inhibition constant.

\section{Results}

3.1. Differential Inhibitory Potential of Maidong's Components towards Different UGT Isoforms. The structures of maidong's components were shown in Figure 1, including ophiopogonin $\mathrm{D}(\mathrm{OD})$, ophiopogonin $\mathrm{D}^{\prime}\left(\mathrm{OD}^{\prime}\right)$, methylophiopogonanone A (MA), methylophiopogonanone B (MB), and ruscorectal (RU). The different structure of all these compounds resulted in the different inhibition potential towards UGT isoforms (Table 1). All the compounds showed little influence towards the activity of UGT1A1, 1A3, 1A7, 1A9, 1A10, 2B4, 2B7, and $2 \mathrm{~B} 15$, with the inhibition percent less than $20 \%$ at $100 \mu \mathrm{M}$. RU exhibits the strongest inhibition towards UGT1A4, with almost $100 \%$ inhibition at $100 \mu \mathrm{M}$ of RU. $100 \mu \mathrm{M}$ of OD and 


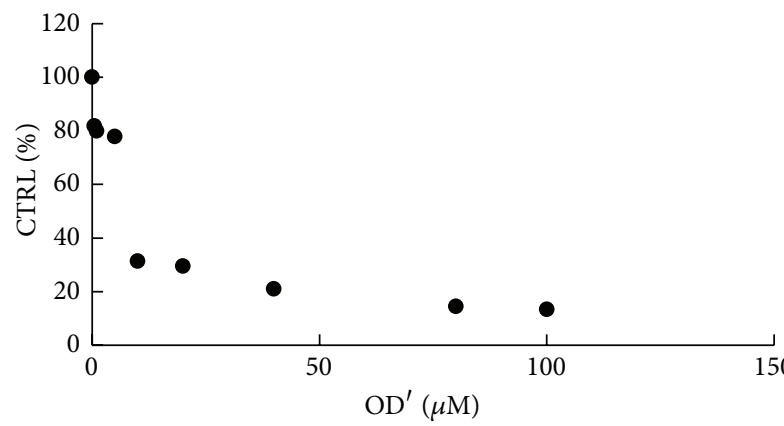

(a)

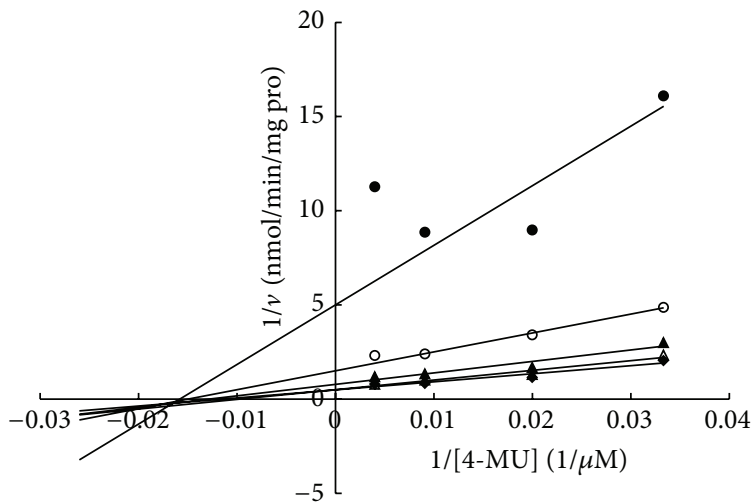

- $\mathrm{OD}^{\prime} 0 \mu \mathrm{M}$

O $\mathrm{OD}^{\prime} 10 \mu \mathrm{M}$

$\triangle \mathrm{OD}^{\prime} 1 \mu \mathrm{M}$

$\Delta \mathrm{OD}^{\prime} 5 \mu \mathrm{M}$

- $\mathrm{OD}^{\prime} 40 \mu \mathrm{M}$

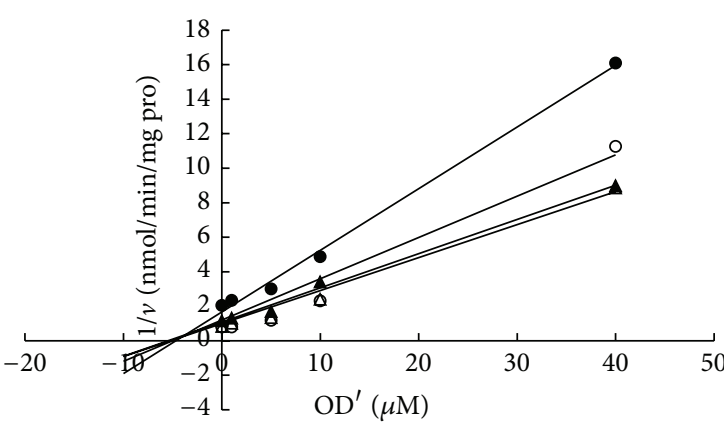

- 4-MU $30 \mu \mathrm{M} \quad \Delta 4-\mathrm{MU} 110 \mu \mathrm{M}$

- $4-\mathrm{MU} 50 \mu \mathrm{M}$

(b)

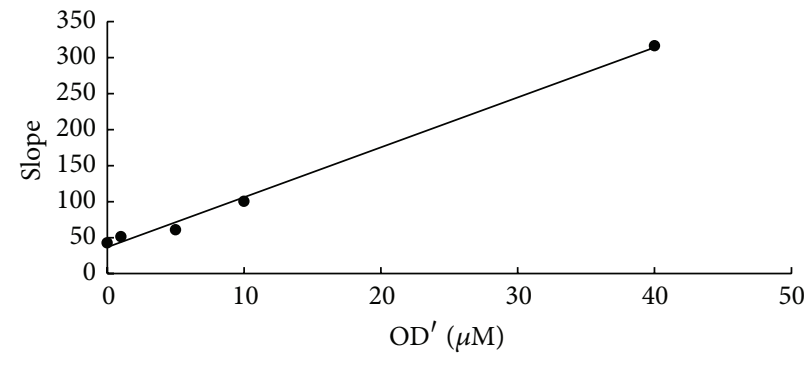

(c)

(d)

FIGURE 4: Determination of inhibition type and parameters $\left(K_{i}\right)$ of $\mathrm{OD}^{\prime}$ towards UGT1A6-catalyzed 4-MU glucuronidation reaction. (a) Dose-dependent inhibition of $\mathrm{OD}^{\prime}$ towards UGT1A6-catalyzed 4-MU glucuronidation. (b) Dixon plot of inhibition of OD' towards UGT1A6catalyzed 4-MU glucuronidation. (c) Lineweaver-Burk plot of inhibition of OD' towards UGT1A6-catalyzed 4-MU glucuronidation. (d) Second plot of inhibition of OD' towards UGT1A6-catalyzed 4-MU glucuronidation. The data point represents the mean value of duplicate experiments.

TABLE 1: Initial screening of the inhibitory effects of ophiopogonin D (OD), ophiopogonin $\mathrm{D}^{\prime}\left(\mathrm{OD}^{\prime}\right)$, methylophiopogonanone A (MA), methylophiopogonanone A (MB), and ruscorectal (RU). $100 \mu \mathrm{M}$ of compounds was selected. The values shown in this table are residual activity which can be calculated using the following equation: $\%$ residual activity $=$ the activity at $100 \mu \mathrm{M}$ of compounds/the activity at $0 \mu \mathrm{M}$ of compounds.

\begin{tabular}{|c|c|c|c|c|c|}
\hline & OD & $\mathrm{OD}^{\prime}$ & MA & $\mathrm{MB}$ & RU \\
\hline UGT1A1 & $77.1 \pm 3.8$ & $81.0 \pm 12.6$ & $31.3 \pm 1.3$ & $27.9 \pm 11.1$ & $41.5 \pm 36.9$ \\
\hline UGT1A3 & $40.7 \pm 0.3$ & $36.5 \pm 2.5$ & $35.5 \pm 6.1$ & $84.2 \pm 22.8$ & $37.2 \pm 18.2$ \\
\hline UGT1A4 & $24.6 \pm 1.6$ & $21.0 \pm 1.6$ & $70.4 \pm 3.8$ & $81.1 \pm 8.0$ & $0.0 \pm 0.0$ \\
\hline UGT1A6 & $12.8 \pm 3.1$ & $12.1 \pm 1.9$ & $67.0 \pm 1.7$ & $65.3 \pm 2.0$ & $65.9 \pm 11.6$ \\
\hline UGT1A7 & $143.5 \pm 8.7$ & $122.2 \pm 1.3$ & $57.5 \pm 10.8$ & $83.4 \pm 5.9$ & $52.4 \pm 7.4$ \\
\hline UGT1A8 & $17.3 \pm 0.9$ & $18.9 \pm 6.6$ & $20.5 \pm 2.1$ & $85.0 \pm 4.5$ & $72.0 \pm 3.0$ \\
\hline UGT1A9 & $135.3 \pm 1.5$ & $133.8 \pm 2.1$ & $38.7 \pm 0.0$ & $68.2 \pm 1.5$ & $110.7 \pm 1.1$ \\
\hline UGT1A10 & $26.5 \pm 1.5$ & $19.8 \pm 3.2$ & $70.0 \pm 9.3$ & $96.9 \pm 7.3$ & $82.0 \pm 5.2$ \\
\hline UGT2B4 & $101.7 \pm 2.6$ & $118.0 \pm 21.8$ & $71.1 \pm 1.5$ & $73.2 \pm 1.2$ & $88.4 \pm 1.6$ \\
\hline UGT2B7 & $85.4 \pm 7.3$ & $77.4 \pm 7.1$ & $23.3 \pm 16.7$ & $82.8 \pm 14.5$ & $124.8 \pm 59.0$ \\
\hline UGT2B15 & $36.9 \pm 0.0$ & $35.5 \pm 1.7$ & $23.8 \pm 2.8$ & $56.1 \pm 9.8$ & $55.5 \pm 19.6$ \\
\hline
\end{tabular}




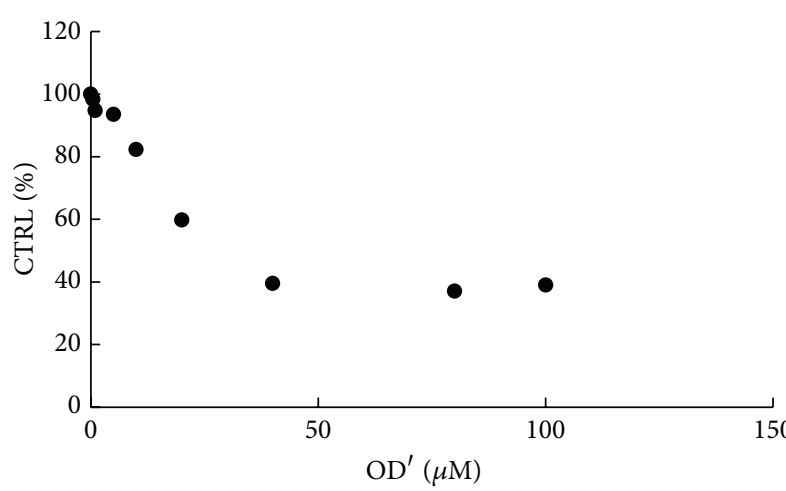

(a)

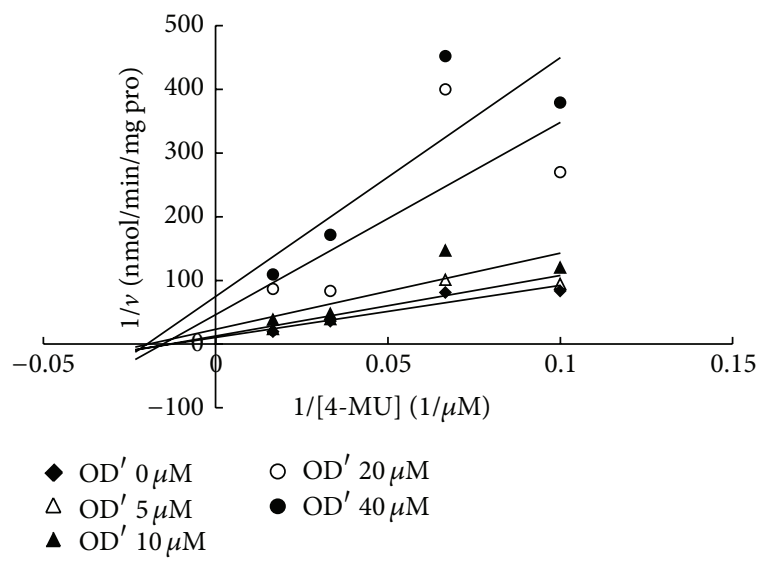

(c)

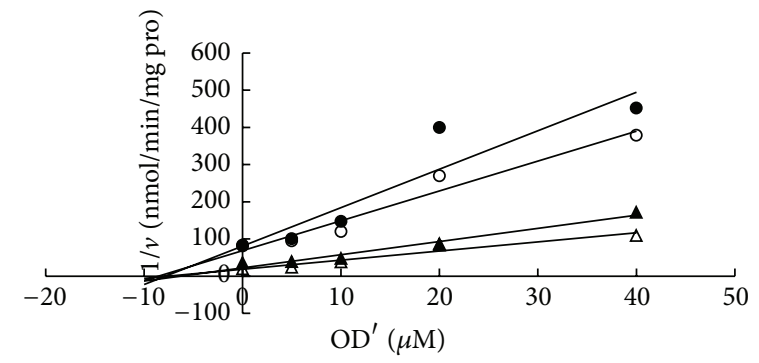

- 4-MU $10 \mu \mathrm{M} \quad \Delta 4-\mathrm{MU} 30 \mu \mathrm{M}$

- 4 -MU $15 \mu \mathrm{M} \quad \Delta 4$-MU $60 \mu \mathrm{M}$

(b)

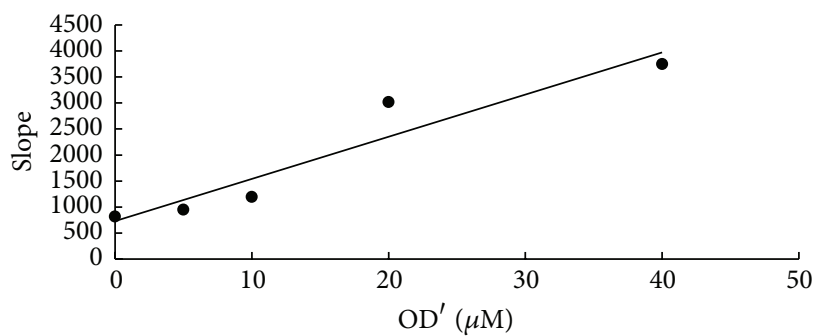

(d)

FIGURE 5: Determination of inhibition type and parameters $\left(K_{i}\right)$ of $\mathrm{OD}^{\prime}$ towards UGT1A10-catalyzed 4-MU glucuronidation reaction. (a) Dose-dependent inhibition of $\mathrm{OD}^{\prime}$ towards UGT1A10-catalyzed 4-MU glucuronidation. (b) Dixon plot of inhibition of OD' towards UGT1A10-catalyzed 4-MU glucuronidation. (c) Lineweaver-Burk plot of inhibition of OD' towards UGT1A10-catalyzed 4-MU glucuronidation. (d) Second plot of inhibition of OD' towards UGT1A10-catalyzed 4-MU glucuronidation. The data point represents the mean value of duplicate experiments.

$\mathrm{OD}^{\prime}$ exerted strong inhibition towards UGT1A6 (87.2\%) and UGT1A8 (82.7\%). The activity of UGT1A6 was also strongly inhibited by $\mathrm{OD}^{\prime}$, with the activity inhibited by $87.9 \%$ at $100 \mu \mathrm{M}$ of $\mathrm{OD}^{\prime}$. The activity of UGT1A10 was inhibited by $80.2 \%$ by $100 \mu \mathrm{M}$ of $\mathrm{OD}^{\prime}$.

\subsection{Inhibition Type and Parameters of Maidong's Compo-} nents towards Representative UGT Isoforms. To furtherly obtain the inhibition kinetic information, we evaluated the inhibition type and parameters of representative inhibition of compounds towards UGT isoforms. The concentrationdependent inhibition was evaluated, and Dixon plot and Lineweaver-Burk plot were employed for elucidation of the inhibition type. Dose-dependent inhibition behaviour can be observed for the inhibition of OD towards UGT1A6 and UGT1A8 (Figures 2(a) and 3(a)), the inhibition of $\mathrm{OD}^{\prime}$ towards UGT1A6 and UGT1A10 (Figures 4(a) and 5(a)), and the inhibition of RU towards UGT1A4 (Figure 6(a)). OD noncompetitively inhibited UGT1A6 and competitively inhibited UGT1A8, as demonstrated by Dixon plot (Figures 2(b) and 3(b)) and Lineweaver-Burk plot (Figures 2(c) and $3(\mathrm{c}))$. $\mathrm{OD}^{\prime}$ noncompetitively inhibited UGT1A6 and UGT1A10 (Figures 4(b), 4(c), 5(b), and 5(c)). Dixon plot (Figure 6(b)) and Lineweaver-Burk plot (Figure 6(c)) showed that RU exerted competitive inhibition towards UGT1A4catalyzed TFP glucuronidation reaction. The inhibition kinetic parameters $\left(K_{i}\right)$ were calculated to be $20.6,40.1,5.3$, 9.0, and $0.02 \mu \mathrm{M}$ for the inhibition of OD towards UGT1A6 and UGT1A8 (Figures 2(d) and 3(d)), the inhibition of $\mathrm{OD}^{\prime}$ towards UGT1A6 and UGT1A10 (Figures 4(d) and 5(d)), and the inhibition of RU towards UGT1A4 (Figure 6(d)).

3.3. Highlight of Components in SMI Showing Strong Inhibition towards UGT Isoforms. We made a relatively complete description on the inhibition potential of SMI major components towards UGT isoforms, including ginsenosides and maidong's and wuweizi's ingredients (Figure 7). The residual activity below $20 \%$ at $100 \mu \mathrm{M}$ was highlighted with red color, 


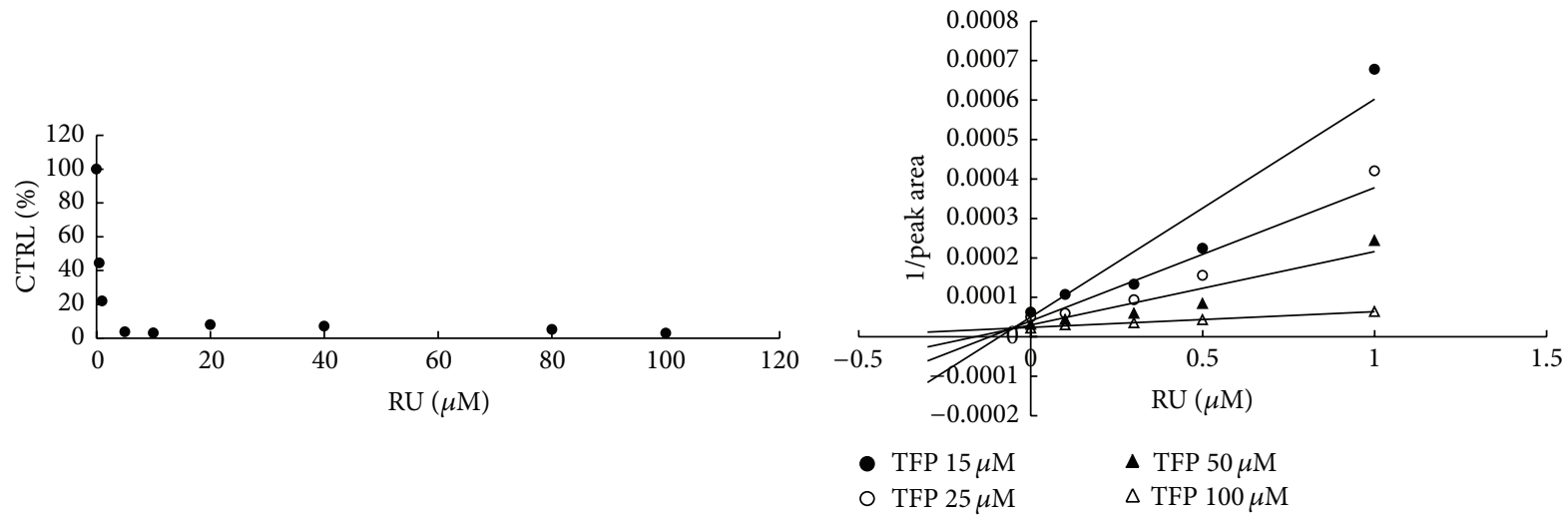

(a)

(b)
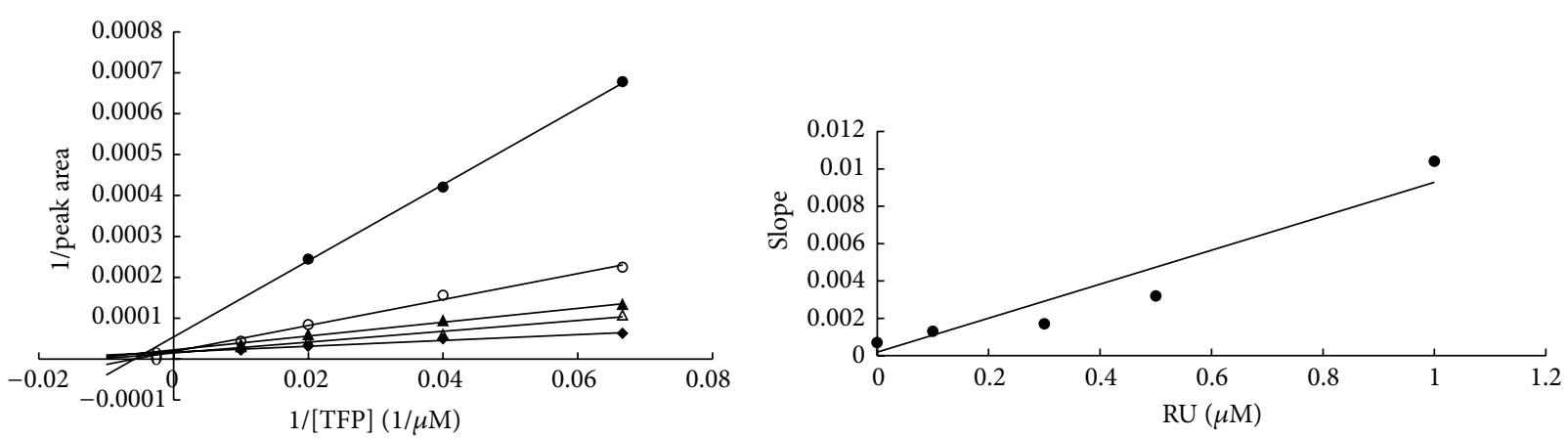

- RU $0 \mu \mathrm{M}$

$\circ \mathrm{RU} 0.5 \mu \mathrm{M}$

$\triangle \mathrm{RU} 0.1 \mu \mathrm{M}$

- RU $1 \mu \mathrm{M}$

$\Delta \mathrm{RU} 0.3 \mu \mathrm{M}$

(c)

(d)

FIGURE 6: Determination of inhibition type and parameters $\left(K_{i}\right)$ of RU towards UGT1A4-catalyzed TFP glucuronidation reaction. (a) Dosedependent inhibition of RU towards UGT1A4-catalyzed TFP glucuronidation. (b) Dixon plot of inhibition of RU towards UGT1A4-catalyzed TFP glucuronidation. (c) Lineweaver-Burk plot of inhibition of RU towards UGT1A4-catalyzed TFP glucuronidation. (d) Second plot of RU's inhibition towards UGT1A4-catalyzed TFP glucuronidation. The data point represents the mean value of duplicate experiments.

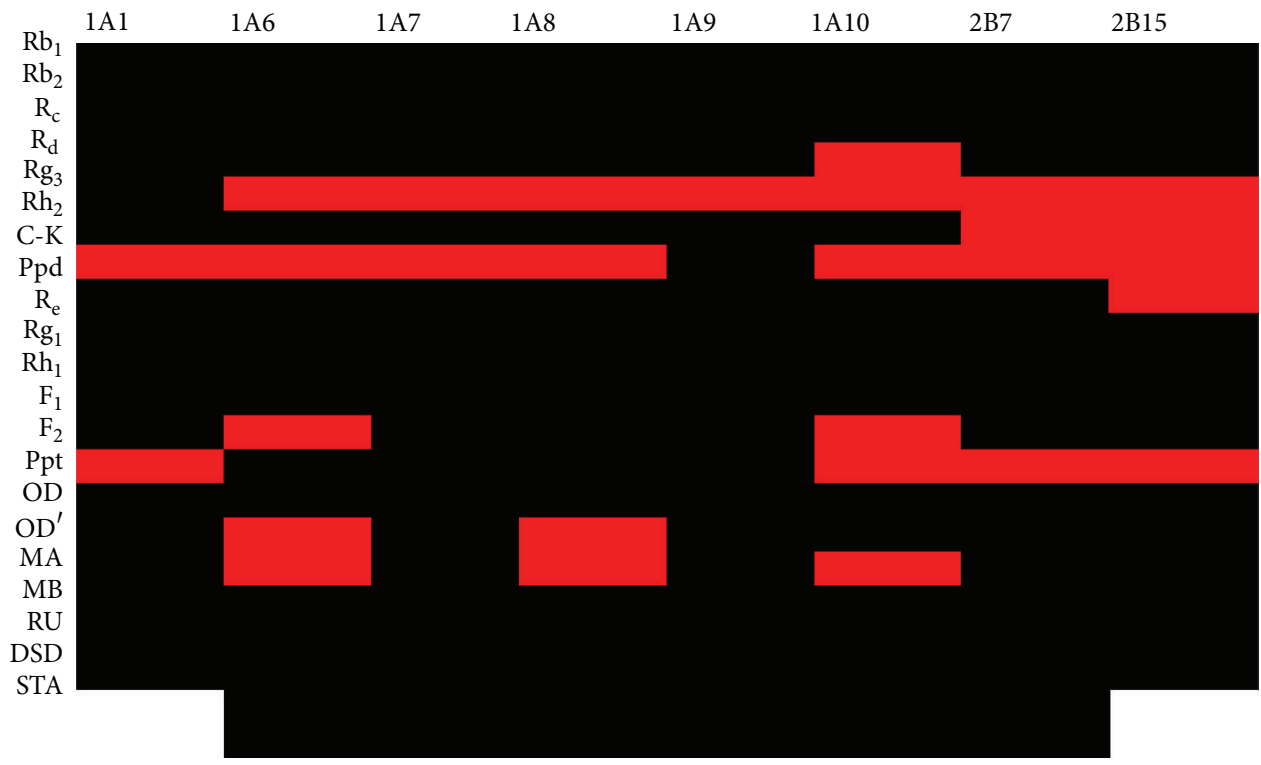

FIGURE 7: Highlight of components in SMI showing strong inhibition towards UGT isoforms. The residual activity below $20 \%$ at $100 \mu \mathrm{M}$ was highlighted with red color, and the residual activity above $20 \%$ at $100 \mu \mathrm{M}$ was highlighted with black color. 
and the residual activity above $20 \%$ at $100 \mu \mathrm{M}$ was highlighted with black color. This figure can clearly tell the readers which components mainly contributed to the inhibition of SMI towards UGT activity.

\section{Discussion}

The safety problem of herbs should be paid much attention, especially when the administration route changes from oral administration to injection because the detoxification function of intestine is avoided. Although searching the targets and substances basis for herbal toxicity is a tremendous job, our previous results encourage our recent study to evaluate the inhibition potential of maidong's major components towards UGT isoforms supposing that UGTs are potential toxicity targets of shengmai injection (SMI).

The information obtained in the present study can help us to obtain a relatively complete inhibition profile of SMI major components towards important UGT isoforms. As is expected, the ingredients of maidong can show the inhibitory potential towards some UGT isoforms, such as the inhibition of UGT1A6 and UGT1A8 by OD, the inhibition of UGT1A6 and UGT1A10 by OD', and the inhibition of UGT1A4 by RU. From the complete inhibitory profile of SMI ingredients towards UGT isoforms, ginsenosides $\mathrm{Rg}_{3}$ and $\mathrm{C}-\mathrm{K}$ are the top two components influencing most UGT isoforms. Additionally, ginsenosides $\mathrm{R}_{\mathrm{d}}, \mathrm{F}_{2}$, ppt, and maidong's components $\mathrm{OD}$ and $\mathrm{OD}^{\prime}$ also significantly contributed to the UGT inhibition by SMI. UGT isoforms play key roles in the metabolism of many endogenous substances. For example, UGT1A1 can catalyze the conjugation reaction of bilirubin $[5,6]$. UGT1A3, UGT1A4, and UGT2B7 can conjugate the bile acids, androgens, and estrogens components through glucuronidation reactions $[10,11]$. Therefore, the influence towards the activity of these UGT isoforms might result in the metabolic disorders of these substances, which might be a potential reason for SMI-induced adverse effects. The in vivo inhibition situation was not performed for SMI due to the little information on the quantity of individual ingredients and the exposure level of these components in plasma.

Many complex factors might affect the explanation of these in vitro data. For example, the activation of drugmetabolizing enzymes (DMEs) through nuclear receptor (e.g., pregnane $\mathrm{X}$ receptor (PXR), peroxisome proliferatoractivated receptor (PPAR), aryl hydrocarbon receptor (Ahr), etc.) is an important reason. Ginsenosides $\mathrm{F}_{2}$ and ppt were demonstrated to exhibit moderate inductive potential towards PXR [12]. The experiment performed by Lee et al. showed that ginsenoside $\mathrm{R}_{\mathrm{f}}$ can regulate lipoprotein metabolism through affecting the activity of PPARalpha [13]. Ginsenosides were also demonstrated to be naturally occurring aryl hydrocarbon receptor (ahr) ligands [14]. Wuweizi ingredients have also been demonstrated to be strong activators towards PXR [15]. All these affected nuclear receptors have been indicated to be able to induce the expression of UGT isoforms [16], which might complicate the explanation of the in vitro inhibition data. Another important factor affecting the explanation of in vitro parameters is the noncomplete understanding of the constitution of SMI.
Although numerous high-grade technologies have been used to elucidate the components, the global characterization of ingredients contained in SMI remains to be challenged [17]. Therefore, all the unknown components in SMI might play an unexpected role towards UGT isoforms-mediated reactions.

In conclusion, the inhibition capability of major components of maidong towards important UGT isoforms was investigated to make relatively complete inhibition profile of SMI ingredients towards the important UGTs. Although many complicated factors might influence the translation of these in vitro results into in vivo situation, all the information obtained in the present study provides a new insight to understand shengmai injection- (SMI-) related drug-drug interaction.

\section{Conflict of Interests}

The authors declare that there is no conflict of interests regarding the publication of this paper.

\section{Acknowledgment}

This work was supported by the National Natural Science Foundation of China (nos. 81202586 and 81202588).

\section{References}

[1] H. Nishida, M. Kushida, Y. Nakajima et al., "Amyloid- $\beta$-induced cytotoxicity of PC-12 cell was attenuated by Shengmai-san through redox regulation and outgrowth induction," Journal of Pharmacological Sciences, vol. 104, no. 1, pp. 73-81, 2007.

[2] B. Xiang, Y. Peng, and Y. G. Jiang, "A report on the analysis of 365 cases of adverse reactions of Chinese patent drugs for heart cerebrovascular diseases," African Journal of Pharmacy and Pharmacology, vol. 7, no. 20, pp. 1267-1271, 2013.

[3] Q. Su and Y. Li, "Interaction between warfarin and the herbal product shengmai-yin: a case report of intracerebral hematoma," Yonsei Medical Journal, vol. 51, no. 5, pp. 793-796, 2010.

[4] C. D. King, G. R. Rios, M. D. Green, and T. R. Tephly, "UDPGlucuronosyltransferases," Current Drug Metabolism, vol. 1, no. 2, pp. 143-161, 2000.

[5] S. D. Zucker, X. Qin, S. D. Rouster et al., "Mechanism of indinavir-induced hyperbilirubinemia," Proceedings of the National Academy of Sciences of the United States of America, vol. 98, no. 22, pp. 12671-12676, 2001.

[6] J. Meza-Junco, Q. S.-C. Chu, O. Christensen et al., "UGT1A1 polymorphism and hyperbilirubinemia in a patient who received sorafenib," Cancer Chemotherapy and Pharmacology, vol. 65, no. 1, pp. 1-4, 2009.

[7] Z.-Z. Fang, Y.-F. Cao, C.-M. Hu et al., "Structure-inhibition relationship of ginsenosides towards UDP-glucuronosyltransferases (UGTs)," Toxicology and Applied Pharmacology, vol. 267, no. 2, pp. 149-154, 2013.

[8] C. Liu, Y. F. Cao, Z. Z. Fang et al., "Strong inhibition of deoxyschizandrin and schisantherin A toward UDP-glucuronosyltransferase (UGT) 1A3 indicating UGT inhibitionbased herb-drug interaction," Fitoterapia, vol. 83, no. 8, pp. 1415-1419, 2012. 
[9] V. Uchaipichat, P. I. Mackenzie, D. J. Elliot, and J. O. Miners, "Selectivity of substrate (trifluoperazine) and inhibitor (amitriptyline, androsterone, canrenoic acid, hecogenin, phenylbutazone, quinidine, quinine, and sulfinpyrazone) "probes" for human UDP-glucuronosyltransferases," Drug Metabolism and Disposition, vol. 34, no. 3, pp. 449-456, 2006.

[10] W. E. Gall, G. Zawada, B. Mojarrabi et al., "Differential glucuronidation of bile acids, androgens and estrogens by human UGT1A3 and 2B7," Journal of Steroid Biochemistry and Molecular Biology, vol. 70, no. 1-3, pp. 101-108, 1999.

[11] N. Sneitz, K. Krishnan, D. F. Covey, and M. Finel, "Glucuronidation of the steroid enantiomers ent-17 $\beta$-estradiol, entandrosterone and ent-etiocholanolone by the human UDPglucuronosyltransferases," Journal of Steroid Biochemistry and Molecular Biology, vol. 127, no. 3-5, pp. 282-288, 2011.

[12] Y.-G. Wang, H.-S. Liu, X.-X. Zhang et al., "Screening of pregnane X receptor activation from ginsenosides," Yao Xue Xue Bao, vol. 48, no. 1, pp. 144-148, 2013.

[13] H. Lee, F. J. Gonzalez, and M. Yoon, "Ginsenoside Rf, a component of ginseng, regulates lipoprotein metabolism through peroxisome proliferator-activated receptor $\alpha$, Biochemical and Biophysical Research Communications, vol. 339, no. 1, pp. 196203, 2006.

[14] Q. Hu, G. He, J. Zhao et al., "Ginsenosides are novel naturallyoccurring aryl hydrocarbon receptor ligands," PLOS ONE, vol. 8, no. 6, Article ID e66258, 2013.

[15] Y. Mu, J. Zhang, S. Zhang et al., "Traditional Chinese medicines Wu Wei Zi (Schisandra chinensis Baill) and Gan Cao (Glycyrrhiza uralensis Fisch) activate pregnane $\mathrm{X}$ receptor and increase warfarin clearance in rats," Journal of Pharmacology and Experimental Therapeutics, vol. 316, no. 3, pp. 1369-1377, 2006.

[16] C. Bigo, S. Caron, A. Dallaire-Théroux, and O. Barbier, "Nuclear receptors and endobiotics glucuronidation: the good, the bad, and the UGT,' Drug Metabolism Reviews, vol. 45, no. 1, pp. 3447,2013

[17] C. Zheng, H. Hao, X. Wang et al., "Diagnostic fragment-ionbased extension strategy for rapid screening and identification of serial components of homologous families contained in traditional Chinese medicine prescription using high-resolution LC-ESI-IT-TOF/MS: shengmai injection as an example," Journal of Mass Spectrometry, vol. 44, no. 2, pp. 230-244, 2009. 


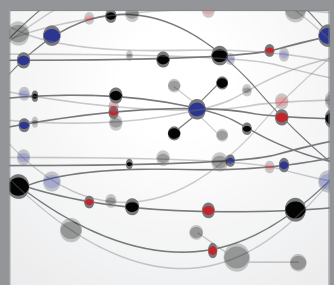

The Scientific World Journal
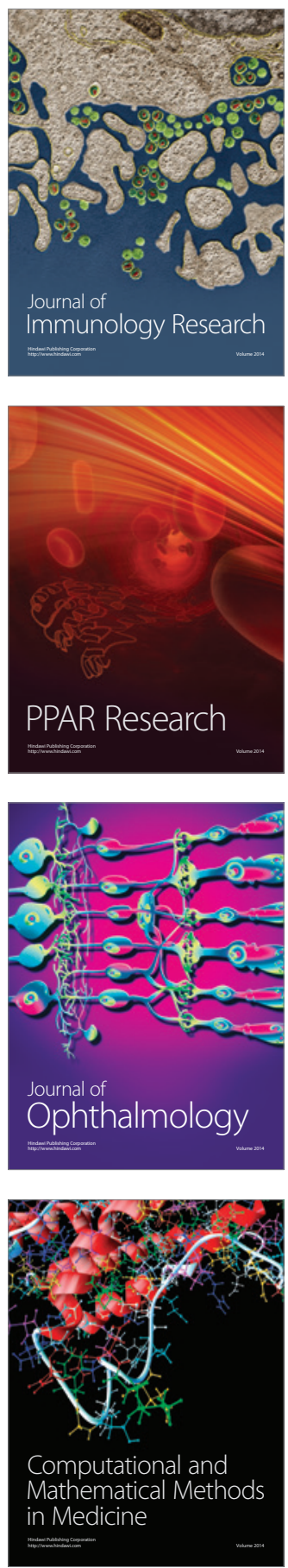

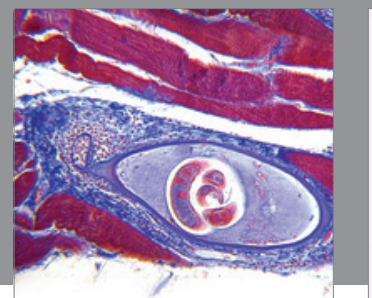

Gastroenterology

Research and Practice
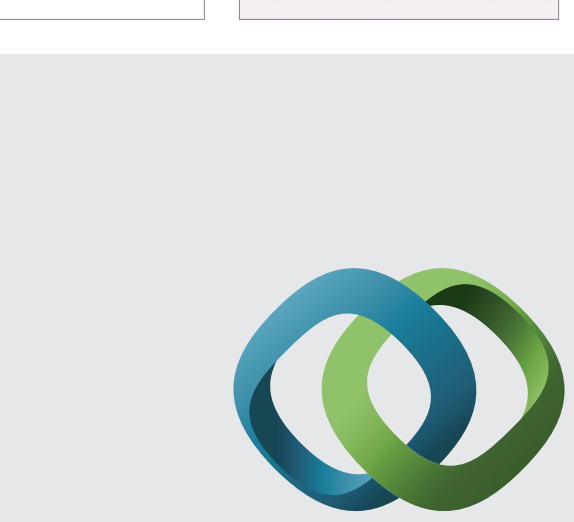

\section{Hindawi}

Submit your manuscripts at

http://www.hindawi.com
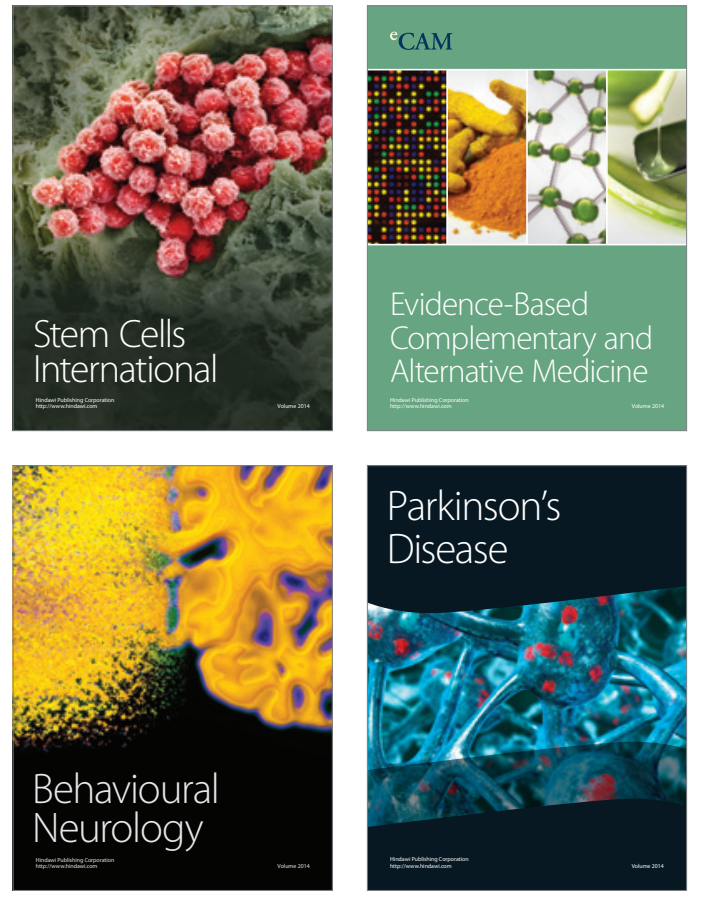
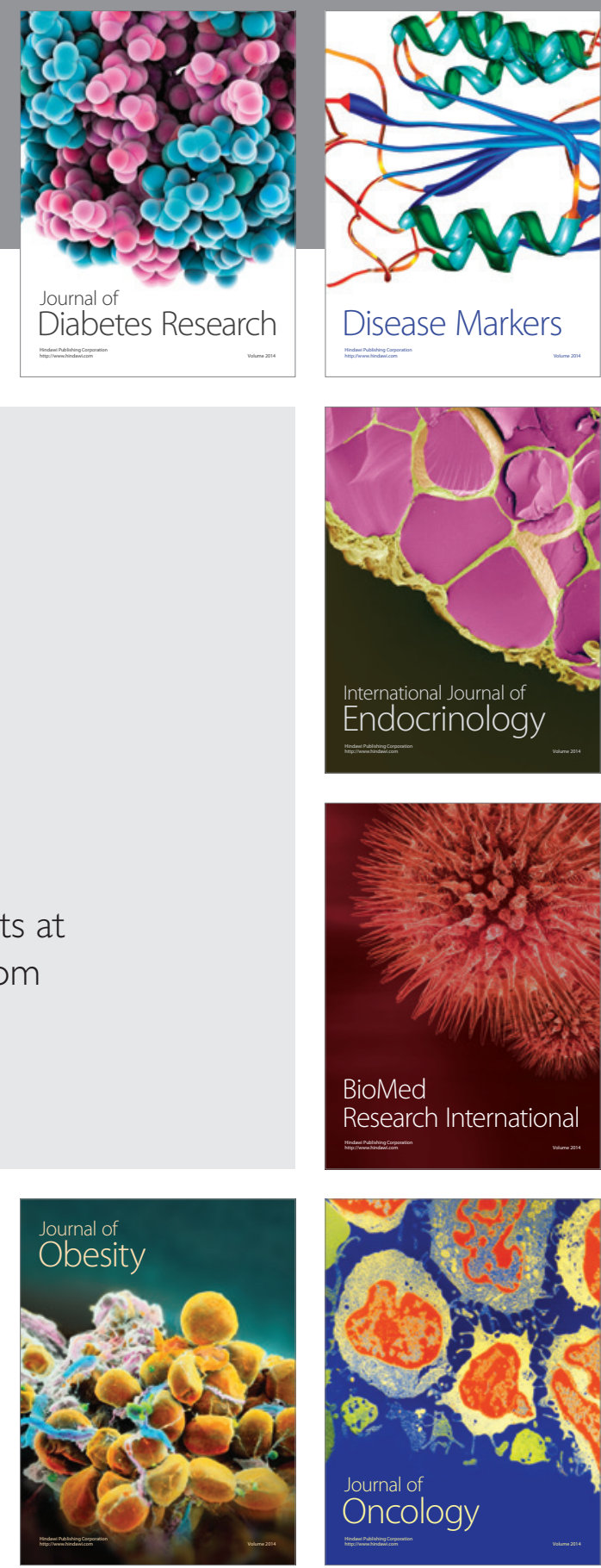

Disease Markers
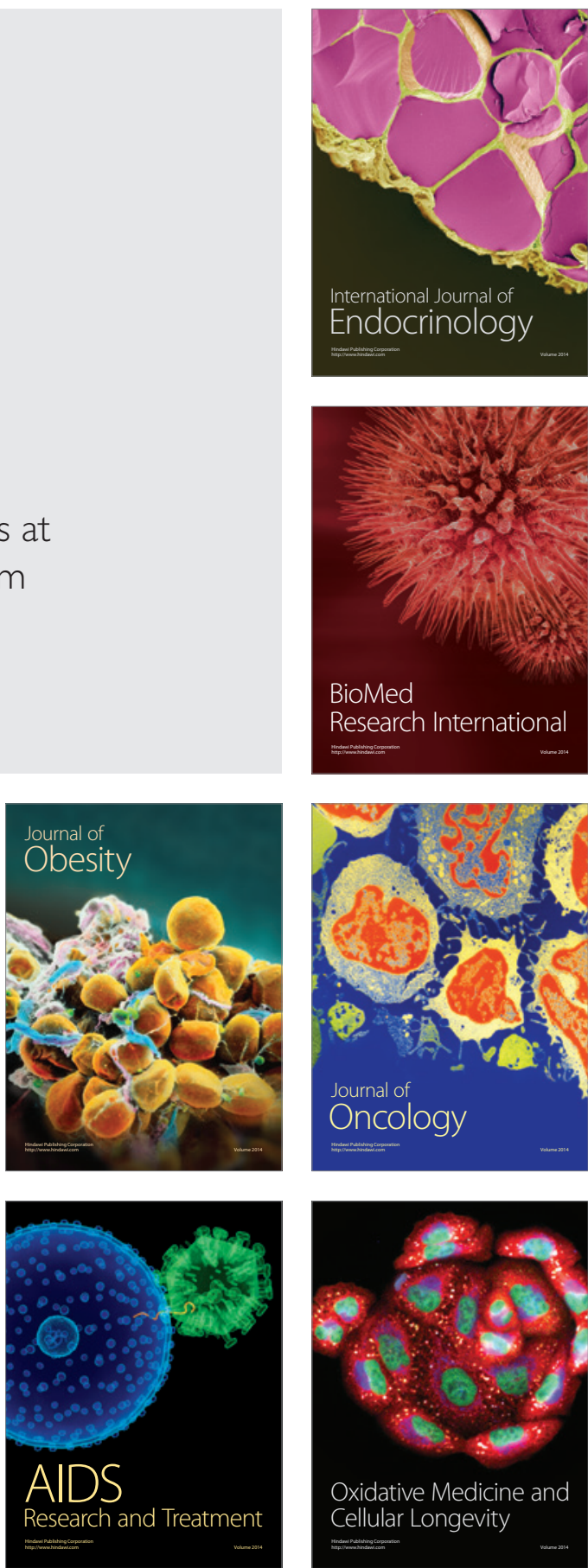